\title{
Ages of Youth Sport Participants - Is Verification a Concern?
}

\section{Manuel J. Coelho-e-Silva ${ }^{1 *}$, Joao Valente-dos-Santos ${ }^{1}$, António J. Figueiredo ${ }^{1}$ and Robert M. Malina ${ }^{2,3}$}

${ }^{1}$ Faculty of Sport Sciences and Physical Education, University of Coimbra, Coimbra, Portugal

${ }^{2}$ Department of Kinesiology and Health Education, University of Texas at Austin, USA

${ }^{3}$ Department of Kinesiology, Tarleton State University, Stephenville, Texas, USA

Cutoff dates for Chronological Age (CA) which establish eligibility for a sport during a season or for a specific competition vary. CA is determined by subtracting date of birth from the cutoff date for the season or competition. The integrity of age-group competitions for youth sport competitions (as well as in other youth activities) is thus based upon the assumption that ages of participants are accurate and official documents (birth certificates, passports) are valid.

Problems with accurate reporting of CA have occurred on a more or less regular basis in youth sports (see Malina [1], for specific citations). A team from the Philippines forfeited the 1992 Little League Baseball championship for allegedly using fraudulent identification papers and overage players, while a team from New York City was disqualified from the 2001 Little League Baseball championship for using a player who was overage. Issues with age falsification extend to other youth competitions, e.g., the $37^{\text {th }}$ Francophone Scrabble World Championships for 14-15 year olds. It also has been alleged that some athletes and/or their parents, sport authorities and agents have falsified CAs. Most recently, it was alleged that CAs of Chinese female artistic gymnasts were falsified to make young athletes chronologically older in order to meet the minimum age requirement for international competitions, 16 years. Although the CAs of Chinese artistic gymnasts were eventually verified as accurate by the International Gymnastic Federation, the issue of age verification in gymnastics continues. Sport agents for a number of youth soccer players who were too young to compete internationally have apparently had birth dates altered on official documents, i.e., modified official documents to have the youngster appear older.

Pressure for success in sport, the international search for talent, and potential for economic gain in elite sport contribute to age falsification or inaccurate reporting. Unfortunately, the fact that talented young athletes are adolescents and in some cases are children is ignored. The youngsters are essentially viewed and treated as commodities by many adults involved in sport.

The International Olympic Committee assembled an expert group to review the current state of age determination in athletes, aiming to provide recommendations for sports governing bodies [2]. The committee report focused to a large extent on variation in biological maturation among individuals during the adolescent years and noted limitations of available methods of assessment for age verification. The committee did not, however, systematically evaluate the reasonably extensive body of literature dealing with the biological maturation, specifically, skeletal and sexual maturation, of young athletes that span almost 50 years [1]

"Bone age" or skeletal age has been used for age verification in international youth soccer [3] and cricket [4] competitions. FIFA has adopted Magnetic Resonance Imaging (MRI) to determine age eligibility in U-17 competitions [5]. Given the popularity of soccer worldwide, the use of Skeletal Age (SA) and MRI in the context of age verification or eligibility determination merits critical evaluation.

\section{Youth Sport and Variation among Adolescents}

By way of background, participation in organized sports declines with increasing age from late childhood through adolescence. This reflects changing interests as youth enter adolescence and the selective or exclusive nature of sport [6,7]. From a biological perspective, adolescence includes the onset of sexual maturation, the growth spurt, and eventual attainment of sexual and skeletal maturity. Individual differences in the timing (when) and tempo (progress) of sexual maturation and the growth spurt, and in the time (CA) of attaining sexual and skeletal maturity are considerable [8].

Indicators of sexual maturation - specifically stages of genital and pubic hair in males and breast and pubic hair in females as described by Tanner [9], have major limitations in the context of age verification. When a youngster is examined, he or she is in a specific stage of sexual maturation; there is no information on when the stage was entered and on how long the individual has been in a stage. Moreover, stages of genital and pubic hair in males and of breast and pubic hair in females, though related, are not equivalent [8].

\section{"Bone Age"}

"Bone age" or Skeletal Age (SA) is an indicator of biological maturity status at the time of observation. SA is used most often to evaluate the level of maturity of the bones of the hand-wrist relative to the reference sample upon which the method of assessment was developed (see below). Assume a youth soccer player has a CA of 15.4 years and an SA of 16.5 years. Although he is chronologically 15.4 years of age, his level of skeletal maturity is equivalent to a boy with a CA of 16.4 years in the reference sample. He is advanced in skeletal maturation relative to the reference. Conversely, a youth player with a CA of 15.4 years may have an SA of 14.2 years. His SA is equivalent to a boy with a CA of 14.2 years in the reference sample; he is late or delayed in skeletal maturation for his CA.

Three methods for the estimation of SA are commonly used. All require a standard radiograph of the hand-wrist skeleton: distal radius and ulna, carpals, metacarpals and phalanges. Two methods are based on samples of American youth (Greulich-Pyle and Fels methods). The third (Tanner-Whitehouse) was initially based on British children, although the most recent version is based on a combined sample of British, Belgian, Spanish, Argentine, American and Japanese youth. The three methods are similar in the principle, but criteria and procedures vary so that SAs assigned with each method are not equivalent.

Details of the methods are beyond the scope of this commentary and are summarized elsewhere $[1,8]$. Although SA has been used as an estimate of CA for sport competitions [1], SA has major limitations for

*Corresponding author: Manuel J. Coelho-e-Silva, Faculty of Sport Sciences and Physical Education, University of Coimbra, Coimbra, Portugal, Tel: 239802770 Fax: 239802779; E-mail: mjcesilva@hotmail.com

Received April 28, 2012; Accepted April 30, 2012; Published May 03, 2012

Citation: Coelho-e-Silva MJ, Valente-dos-Santos J, Figueiredo AJ, Malina RM (2012) Ages of Youth Sport Participants - Is Verification a Concern? J Sports Med Doping Stud 2:e107. doi:10.4172/2161-0673.1000e107

Copyright: (C) 2012 Coelho-e-Silva MJ, et al. This is an open-access article distributed under the terms of the Creative Commons Attribution License, which permits unrestricted use, distribution, and reproduction in any medium, provided the original author and source are credited. 
the purpose of age verification. The vast majority of youth athletes are adolescents so that focus is on this age range. The Greulich-Pyle and Fels methods use the carpals, radius, ulna and short bones in assessing SA. The most recent version of the Tanner-Whitehouse method (TW3) has two separate protocols, one limited to seven carpal bones and the other based on the radius, ulna and metacarpals and phalanges of the first, third and fifth digits. The former provides a Carpal SA while the latter provides a Radius-Ulna-Short Bone (RUS) SA.

If an individual has attained skeletal maturity, an SA is not assigned; the individual is simply noted as skeletally mature since in cross-sectional samples (as in athletes or medico-legal cases) it is not known when maturity was attained. With the Greulich-Pyle method, age at maturity is somewhat variably defined. Differences in plates for girls at SAs of 16, 17 and 18 years and for boys at 18 and 19 years are negligible. With the Fels method, an SA of 18.0 years indicates maturity in both sexes. With the TW3 RUS method, an SA of 15.0 years in girls and 16.5 years in boys are associated with skeletal maturity. Carpal maturity is attained earlier than RUS maturity in TW3.

A factor underlying the discrepancy between the Fels and TW3 methods relates to the final stage of maturation of the radius and ulna. With the Tanner-Whitehouse protocol, the criteria for the final stage of maturation of the distal radial and ulnar epiphyses are that "...fusion of the epiphysis and metaphysis has begun." The time lag between the onset and completion of epiphyseal union is not considered. In contrast, the Fels method has specific criteria from beginning through complete union. Note that the radius is the last bone of the handwrist complex to reach maturity (i.e., complete union). With the TW3 protocol, many youth are classified as skeletally mature even though the process of epiphyseal union is not fully completed.

Although the Greulich-Pyle method is widely used, its application is variable. The protocol calls for the assessment of the SA of each individual bone relative to standard plates in the atlas; the median of the SAs is then the SA of the individual. In practice, however, the protocol is applied by comparing the hand-wrist radiograph of an individual as a whole to the standard plates in the atlas; the SA of the plate to which it most closely matches is then assigned as the SA of the individual. This practice is problematic as variation in level of maturity among individual bones is overlooked; there is also a need for interpolation between standard plates.

The preceding reflects methodological issues. A more problematic issue is variation in SA within a CA group. Normal variation in SA is generally accepted as plus/minus three standard deviations, except as maturity is approached [10]. Standard deviations for SA within single year CA groups $10-17$ years range from approximately 0.7 to 1.4 years with the Greulich-Pyle and Fels methods [1]. Standard deviations approximate 1 year from childhood through adolescence for TannerWhitehouse RUS SAs, but age-specific values were not reported [11]. Allowing for differences among methods, it is likely that the range of variation in SA within a CA group can exceed 4 or perhaps 5 years. Such variation limits the utility of SA as a means for verifying CA in youth sport competitions.

Of specific relevance for adolescent male athletes in many sports, use of SA to verify CA will eliminate a good number of age-eligible boys. Adolescent male athletes in a variety of sports (with the exception of artistic gymnastics) tend to be, on average, advanced in skeletal maturity; they are also advanced in sexual maturity and timing of the growth spurt [8]. Although there is variation among methods of assessment, significant numbers of adolescent male athletes 14-17 years of age are skeletally mature and would be eliminated from agegroup competitions [1].
Although the use of SA for CA verification in age group competitions for female athletes is less often discussed, it received considerable discussion in the context of artistic gymnastics during the Beijing Olympic Games in 2008. Adolescent artistic gymnasts tend to be on average delayed in SA relative to their CA. The lateness tends to increases with CA [1]. At present, gymnasts must have attained their $16^{\text {th }}$ birthday to be eligible for Olympic competition. Unfortunately, SA data for artistic gymnasts are reported as means and standard deviations so that the range of variation in specific CA groups cannot be ascertained [1]. Nevertheless, data for participants 13-19 years from the 1987 World Championship, RUS SAs based on the earlier version (TW2) provide insights of relevance to age verification. Among 14 and 15 year old female gymnasts, respectively, 7 of 44 and 10 of 44 were skeletally mature. Although age-ineligible for current competition (16.0 years), there are skeletally mature and based on this would be eligible. On the other hand, many age-eligible gymnasts (16+ years) would be declared ineligible for competition because their SAs were less than 16.0 years (i.e., not skeletally mature). In the Rotterdam sample, 19 of 31 gymnasts 16 years and 16 of 38 gymnasts 17 years of age had SAs less than 16.0 years. In fact, 6 of 24 gymnasts $18-19$ years had SAs less than 16.0 years [1].

\section{MRI of the Distal Radius}

Use of fusion or union of the distal radial epiphysis and diaphysis as assessed with MRI has been used as an alternative for age verification in U-17 soccer competitions [5]. Although the protocol described stages of union, focus is on the number of completely used (mature) players within CA groups. The protocol is apparently aimed at identifying mature and presumably overage players; it does not provide an SA.

Of relevance to the present discussion, a significant number of age-eligible participants in several FIFA sponsored U-17 international soccer competitions (in Finland, Japan, Peru and Singapore) presented complete radial fusion [5]. More specifically, among 8 players 14 years of age, 3 presented complete union; among 27 players 15 years, 4 presented complete union. Among older players, 18 of 85 players 16 years and 17 of 66 players 17 years of age presented complete union of the distal radial epiphysis. Although use of MRI in FIFA U-17 competitions is set in the context of fair play, what is fair about denying an age-eligible boy the opportunity to compete because he is advanced in skeletal maturation?

\section{No Method is Foolproof}

Clearly CA verification presents potential problems for organizers of youth sport competitions. Neither SA nor MRI assessment of distal radial union provides an accurate estimate of $\mathrm{CA}$. Both methods have the potential to eliminate a significant number of CA eligible male athletes. Although data are not as extensive for female athletes, similar problems apply. The data for females are confounded in part by earlier or advanced maturation of females compared to males and by the trend for later maturing females to be successful in some sports. An additional confounding factor in the use of SA or MRI for age verification in international youth sport competitions is ethnic variation in skeletal maturation [1].

Another issue that needs consideration is the fact that births of relatively large numbers of children in some developing countries are not registered, i.e., they do not have an official birth date or birth certificate [12]. The issue is confounded if parents wait a year or two, and perhaps more, before they register their child. The late date of registration may become the child's official "birth date". The child is thus classified as younger than he/she really is. 
Citation: Coelho-e-Silva MJ, Valente-dos-Santos J, Figueiredo AJ, Malina RM (2012) Ages of Youth Sport Participants - Is Verification a Concern? J Sports Med Doping Stud 2:e107. doi:10.4172/2161-0673.1000e107

\section{References}

1. Malina RM (2011) Skeletal age and age verification in youth sport. Sports Med 41: $925-947$.

2. Engebretsen L, Steffen K, Bahr R, Broderick C, Dvorak J, et al. (2010) The International Olympic Committee Consensus statement on age determination in high-level young athletes. Br J Sports Med 44: 476-484.

3. CNN Sports Illustrated (2001) 'Cheating does not pay': Asia bans teams, players for over-age infractions.

4. Asian Cricket Council (2007) U-15 ACC elite age-verification results issued.

5. Dvorak J, George J, Junge A, Hodler J (2007) Application of MRI of the wrist for age determination in international U-17 soccer competitions. $\mathrm{Br} \mathrm{J}$ Sports Med 41: 497-500.

6. Malina RM (2009) Children and adolescents in the sport culture: The overwhelming majority to the select few. J Exerc Sci Fit 7: S1-S10.
7. Malina RM (2010) Early sport specialization: roots, effectiveness, risks. Cur Sports Med Rep 9: 364-371.

8. Malina RM, Bouchard C, Bar-Or O (2004) Growth, maturation, and physical activity. (2ndedn) Champaign, IL: Human Kinetics.

9. Tanner JM (1962) Growth at adolescence. ( $2^{\text {nd }}$ ed.) Oxford: Blackwell.

10. Roche AF, Chumlea CW, Thissen D (1988) Assessing the Skeletal Maturity of the Hand-Wrist: Fels Method. Springfield, IL: CC Thomas.

11. Tanner JM, Healy MJR, Goldstein H, Cameron N (2001) Assessment of Skeletal Maturity and Prediction of Adult Height (TW3 Method). ( $3^{\text {rd }}$ ed.) London: Saunders.

12. UNICEF (2009) Progress for children: a report card on child protection. UNICEF Division of Communications, New York. 